\title{
Post-conditioning with sevoflurane induces heme oxygenase-1 expression via the PI3K/Akt pathway in lipopolysaccharide-induced acute lung injury
}

\author{
SHUANGPING ZHAO ${ }^{1}$, JIAO WU ${ }^{2}$, LEMENG ZHANG ${ }^{1}$ and YUHANG AI ${ }^{1}$ \\ ${ }^{1}$ Department of Critical Care Medicine, Hunan Xiangya Hospital, Central South University, Changsha, Hunan 410008; \\ ${ }^{2}$ Hunan Provincial Tumor Hospital, Changsha, Hunan 410013, P.R. China
}

Received July 24, 2013; Accepted March 3, 2014

DOI: $10.3892 / \mathrm{mmr} .2014 .2094$

\begin{abstract}
The aim of the present study was to explore the regulatory mechanism of heme oxygenase-1 (HO-1) expression induced by sevoflurane (Sevo) in lipopolysaccharide (LPS)-induced acute lung injury (ALI). Sprague-Dawley rats were divided randomly into six groups: (A) Control, (B) 2.4\% Sevo only, (C) LY294002 (PI3K inhibitor) only, (D) LPS + 2.4\% Sevo, (E) LY294002 + LPS + 2.4\% Sevo and (F) LPS only. The pathological changes in wet/dry weight ratio (W/D), the activities of superoxide dismutase, myeloperoxidase (MPO), malondialdehyde, and $\mathrm{HO}-1$, as well as the expression of intercellular adhesion molecule (ICAM-1), HO-1, phospho-phosphatidylinositol 3-kinase (pPI3K) and phospho-Akt (pAkt) were recorded. Sevo post-conditioning was able to effectively protect from ALI with decreasing pathomorphological scores, MPO activity, W/D and the mRNA and protein expression levels of ICAM-1. Sevo promotes HO-1 expression via the PI3K/protein kinase B (PI3K/Akt) pathway with activation of $\mathrm{pPI} 3 \mathrm{~K}$ and $\mathrm{pAkt}$. Inhibition of the PI3K/Akt pathway by LY294002 partly eliminates the protective effects of Sevo. It is concluded that Sevo post-conditioning has a vital role in inducing the upregulation of $\mathrm{HO}-1$ expression via the $\mathrm{PI}$ KK/Akt pathway to alleviate ALI.
\end{abstract}

\section{Introduction}

Epithelial and endothelial cell perturbation and inflammatory cell influx are indicative of acute lung injury (ALI), which is a diffuse heterogeneous lung injury that leads to hypoxemia, non-cardiogenic pulmonary edema, low lung compliance and widespread capillary leakage. As an inducible enzyme for the

Correspondence to: Dr Yuhang Ai, Department of Critical Care Medicine, Hunan Xiangya Hospital, Central South University, No. 87 Xiangya Road, Changsha, Hunan 410008, P.R. China E-mail: ayhicu1978@sina.com

Key words: sevoflurane, acute lung injury, heme oxygenase-1, phosphatidylinositol 3-kinase/protein kinase B rate-limiting step in the conversion of heme into biliverdin, carbon monoxide (CO) and free iron, heme oxygenase-1 (HO-1) is also widely accepted as a cellular defense mechanism against harmful or noxious stimuli $(1,2)$. A previous study found that HO-1 expression prevents rat livers from ischemia/reperfusion (I/R) injury when using anesthetic sevoflurane (Sevo) clinically (3). Inhalation of Sevo prior to ischemia increases the activity of HO-1 in the lung and protects from lung I/R injury in rats (4). I/ $\mathrm{R}$ injury is an important model of oxidant-mediated ALI (5). Therefore, Sevo may have an important role in ALI protection by upregulation of HO-1 expression. However, the regulatory mechanism of HO-1 expression is complex and is involved in numerous intracellular signal transduction pathways, including the phosphatidylinositol 3-kinase/protein kinase B (PI3K/Akt) pathway (6).

$\mathrm{PI} 3 \mathrm{~K}$ is a lipid kinase and generates phosphatidylinositol (3,4,5)-trisphosphate (PIP3). PIP3 is a second messenger essential for the translocation of Akt to the plasma membrane where it is phosphorylated and has a pivotal role in fundamental cellular functions, including cell proliferation and survival by phosphorylating a variety of substrates (7). A previous report demonstrated that $\mathrm{HO}$-1-induced protection against hypoxia/reoxygenation is dependent on biliverdin reductase and its interaction with the PI3K/Akt pathway (8). In addition, the PI3K/Akt signaling pathway also has been found to be involved in HO-1 upregulated expression induced by epigallocatechin, 4-methylcatechol and schisandrin (9-11). Therefore, in the present study, the role of the PI3K/Akt regulatory pathway in the upregulated expression of HO-1 induced by Sevo used for the treatment of lipopolysaccharide (LPS)-induced ALI was explored.

\section{Materials and methods}

Animal preparation. The animal protocol was approved by the Institutional Animal Care and Use Committee at Central South University (Changsha, China). Animal models were established according to the methods of Sun et al (12), Crawford et al (13) and Coimbra et al (14). Rats were obtained from Beijing Vital River Laboratory Animal Technology Co., Ltd. (Beijing, China). They were housed with free access to food and water under an automatic 12-h light/12-h dark 
cycle at a temperature of $24^{\circ} \mathrm{C}$ and a humidity of 50-60\%. Following $12 \mathrm{~h}$ fasting, forty-eight male Sprague-Dawley (SD) rats, 220 to $280 \mathrm{~g}$, were anesthetized by intraperitoneal injection of $20 \%$ urethane $(1 \mathrm{~g} / \mathrm{kg})$ and placed in a supine position to implement tracheotomy. The animals were intubated with a homemade tube and mechanically ventilated for $390 \mathrm{~min}$ (with $8 \mathrm{ml} / \mathrm{kg}$ tidal volume and 65-70 times/min respiratory frequency). Right femoral artery intubation was used to measure arterial pressure and blood collection, while the left femoral vein was used for normal saline (NS) and drug administration.

Following ventilation for 20 min with stable conditions, rats were randomized to concurrently receive NS, $2.4 \%$ Sevo (Baxter Healthcare Corporation, Deerfield, IL, USA), delivered through a rodent ventilator (ALC-V8, Shanghai Alcott Biotech Co., Shanghai, China) (13) or LY294002 (a PI3K inhibitor; $0.3 \mathrm{mg} / \mathrm{kg}$, intravenously; Cell Signaling Technology, Inc., Danvers, MA, USA) (15) to yield the following experimental groups: Control, $2.4 \%$ Sevo only, LY294002 only, LPS (5 mg/kg, intravenously; SigmaAldrich, St. Louis, MO, USA) + 2.4\% Sevo, LY294002 + LPS $+2.4 \%$ Sevo and LPS only (Fig. 1). Sevo, delivered by gaseous admixture (oxygen) at a concentration of $2.4 \%$ via a calibrated vaporizer, was administered via an endotracheal tube. The inspired oxygen and Sevo concentrations were also monitored continuously through the anesthetic agent monitor (Datex Instrumentarium, Helsinki, Finland). The rectal temperature of all the rats were maintained between $37-38^{\circ} \mathrm{C}$ by incandescent bulb heating. Following $6 \mathrm{~h}$ drug treatment or saline, all the rats were sacrificed by exsanguination under anesthesia. The tissue samples of the left lung were immediately immersed in liquid nitrogen and stored at $-80^{\circ} \mathrm{C}$ for subsequent activity analysis.

Wet/dry (W/D) lung weight ratios. A total of $100 \mathrm{mg}$ was excised from the right middle lobe, rinsed briefly in PBS, blotted and weighed to obtain the 'wet' weight. Lungs were then dried in an oven at $80^{\circ} \mathrm{C}$ for $48 \mathrm{~h}$ to obtain the 'dry' weight.

MPO, MDA and SOD activity. As an indicator of migration of polymorphonuclear neutrophils in lung tissue, the activity of myeloperoxidase (MPO) was measured with a EnzChek ${ }^{\circledR}$ Myeloperoxidase Activity Assay kit (Thermo Fisher Scientific, Waltham, MA, USA) according to the manufacturer's instructions. The malondialdehyde (MDA) assay was performed to evaluate the severity of lipid peroxidation injury by the thiobarbituric acid (TBA) colorimetric method using an MDA Assay kit (Jiancheng Corp., Nanjing, China). This assay is based on the reaction of MDA with TBA, forming stable thiobarbituric acid-reactive substances, which has a maximum absorption at $532 \mathrm{~nm}$. Superoxide dismutase (SOD) activity was determined using an SOD assay kit (Jiancheng Corp.).

Histological analysis. The pathological changes of lung tissue were examined by hematoxylin and eosin staining under an optical microscope (CHBS microscope; Olympus, Tokyo, Japan). The right upper lobe was dissected, fixed with $4 \%$ paraformaldehyde, dehydrated, embedded in paraffin, sectioned and stained. The degree of lung injury was assessed

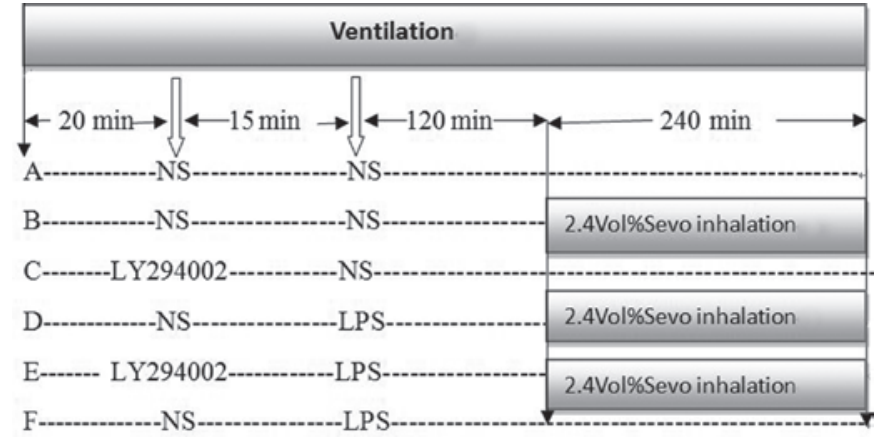

Figure 1. Flowchart of experiment.

using the scoring system described by Al-Amran et al (16). Each parameter was graded on a scale of 0-3, as follows: i) 0 , Alveolar septae. 0, Septae thin and delicate; 1 , congested alveolar septae in $<1 / 3$ of the field; 2 , congested alveolar septae in $1 / 3-2 / 3$ of the field; 3 , congested alveolar septae in $>2 / 3$ of the field. ii) Intra-alveolar cell infiltrates. $0,<5$ intra-alveolar cells per field; 1, 5-10 intra-alveolar cells per field; 2 , 10-20 intra-alveolar cells per field; 3, >20 intra-alveolar cells per field. iii) Alveolar hemorrhage. 0, No hemorrhage; $1,>5$ erythrocytes per alveolus in $1-5$ alveoli; $2,>5$ erythrocytes in 5-10 alveoli, 3, $>5$ erythrocytes in $>10$ alveoli. The total lung injury score was calculated by adding the individual scores for each category. The histological sections were evaluated by a pathologist without prior knowledge of the treatment administered to the animals.

Quantitative polymerase chain reaction $(q P C R)$ and western blot analysis. The cDNA was generated from $2 \mu \mathrm{g}$ total RNA isolated with TRIzol reagent (Invitrogen Life Technologies, Carlsbad, CA, USA) using the RT-PCR kit (MBI, Fermentas, Vilnius, Lithuania) with Oligo (dT) primers and GAPDH as the control. The PCR primers used were: HO-1 forward, 5'-ATGGAGCGCCCACAGCTCGA-3' and reverse, 5'-CTCCAGAGTGTTCATGCGAG-3'; and GAPDH forward, 5'-CAGCAATTTTCAGTGTCAGAAGCT-3' and reverse, 5'-TCATCCTGTCCTTGAGGCAGTAT-3'. Western blot analysis was performed as described previously (17). Immunoblotting was performed with the following antibodies: Rabbit anti-mouse HO-1 (Merck, Darmstadt, Germany), rabbit anti-mouse PI3K-P110 $\alpha$, rabbit anti-mouse phospho-PI3K (pPI3K), rabbit anti-mouse Akt, rabbit anti-mouse phospho-Akt (pAkt) (all Cell Signaling Technology, Inc.), and IRDye 800 goat anti-rabbit (LI-COR Biosciences, Lincoln, NE, USA). Blots were scanned using the Odyssey infrared imaging system (LI-COR Biosciences). GAPDH and $\beta$-actin were used as a control gene and proteins for normalization using the AlphaImager 200 Digital Imaging System (Alpha Innotech, San Leandro, CA, USA).

Statistical analysis. All data were analyzed using SPSS 13.0 (SPSS Inc., Chicago, IL, USA) and the data are expressed as the mean \pm standard deviation. A one-way analysis of variance was used for comparisons between groups. Post-hoc comparisons were performed using a least significant difference test or Dunnett's T3 test. $\mathrm{P}<0.05$ was considered to indicate a statistically significant difference. 
Table I. Comparison of the W/D ratio, SOD, MDA and MPO activity in each group.

\begin{tabular}{lcccr}
\hline $\begin{array}{l}\text { Group } \\
(\mathrm{n}=8)\end{array}$ & $\begin{array}{c}\text { W/D } \\
\text { ratio }\end{array}$ & $\begin{array}{c}\text { SOD activity } \\
(\mathrm{U} / \text { gprot })\end{array}$ & $\begin{array}{c}\text { MDA activity } \\
\text { (nmol/gprot) }\end{array}$ & $\begin{array}{c}\text { MPO activity } \\
(\mathrm{U} / \text { gprot })\end{array}$ \\
\hline $\mathrm{A}$ & $4.11 \pm 0.35$ & $19.55 \pm 1.62$ & $1.50 \pm 0.27$ & $5.12 \pm 0.41$ \\
$\mathrm{~B}$ & $4.24 \pm 0.44$ & $20.07 \pm 1.96$ & $1.67 \pm 0.45$ & $5.28 \pm 0.56$ \\
$\mathrm{C}$ & $4.04 \pm 0.28$ & $19.79 \pm 2.04$ & $1.75 \pm 0.43$ & $5.23 \pm 0.53$ \\
$\mathrm{D}$ & $4.84 \pm 0.39^{\mathrm{a}}$ & $15.32 \pm 1.03^{\mathrm{a}}$ & $2.25 \pm 0.30^{\mathrm{a}}$ & $6.64 \pm 0.74^{\mathrm{a}}$ \\
$\mathrm{E}$ & $4.96 \pm 0.20^{\mathrm{a}}$ & $12.66 \pm 1.97^{\mathrm{a}, \mathrm{b}}$ & $2.65 \pm 0.53^{\mathrm{a}, \mathrm{c}}$ & $7.81 \pm 0.74^{\mathrm{a}, \mathrm{b}}$ \\
F & $4.85 \pm 0.40^{\mathrm{a}}$ & $10.28 \pm 0.99^{\mathrm{a}, \mathrm{b}}$ & $3.67 \pm 0.49^{\mathrm{a}, \mathrm{b}}$ & $8.18 \pm 0.81^{\mathrm{a}, \mathrm{b}}$ \\
\hline
\end{tabular}

${ }^{\mathrm{a}} \mathrm{P}<0.05$, compared with group A. ${ }^{\mathrm{b}} \mathrm{P}<0.05$, compared with group D. ${ }^{\mathrm{c}} \mathrm{P}<0.05$, compared with group F. A, control; B, $2.4 \%$ Sevo; C, LY294002 (phosphatidylinositol 3-kinase inhibitor); D, LPS + 2.4\% Sevo; E, LY294002 + LPS + 2.4\% Sevo; F, LPS; W/D, wet/dry weight; MPO, myeloperoxidase; SOD, superoxide dismutase; MDA, malondialdehyde.
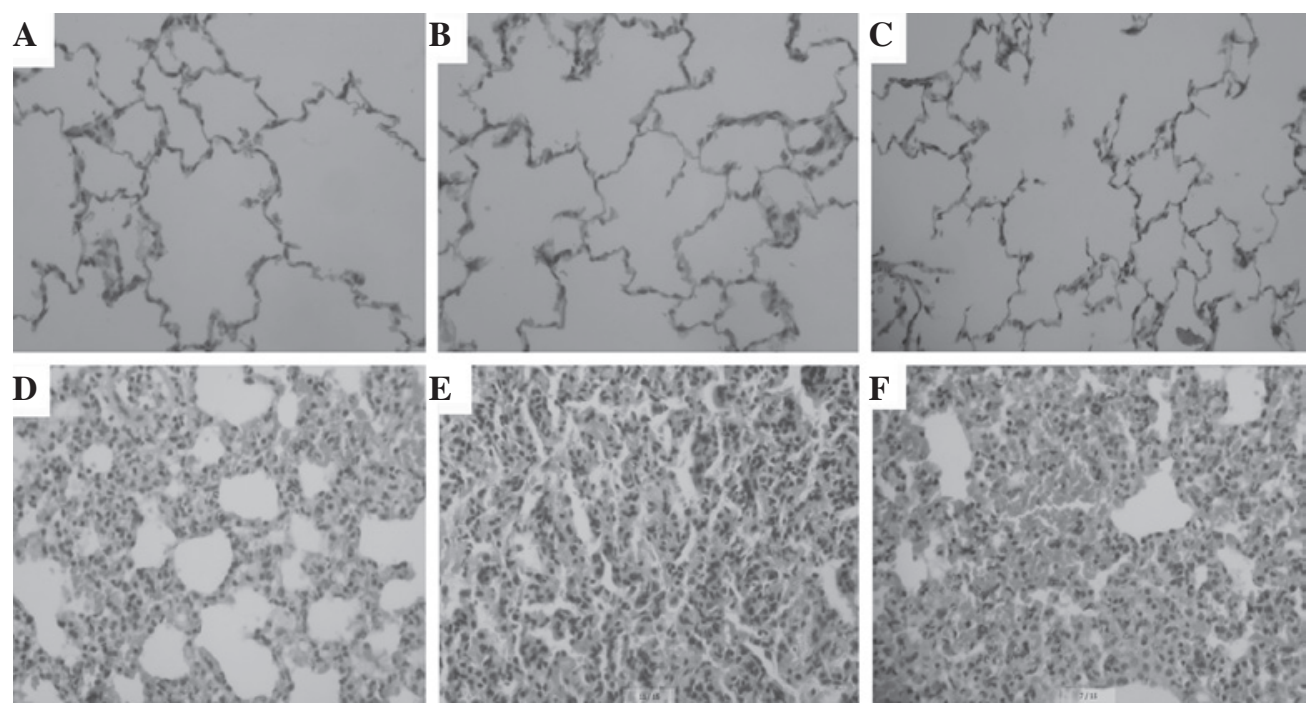

Figure 2. Pathological changes of lung in acute injury with different treatment in each group. (A) control; (B) 2.4\% Sevo; (C) LY294002 (phosphatidylinositol 3-kinase inhibitor); (D) LPS + 2.4\% Sevo; (E) LY294002 + LPS + 2.4\% Sevo; (F) LPS. Magnification, x200. Sevo, sevofluorane; LPS, lipopolysaccharide.

\section{Results}

LPS injection induces lung tissue damage with increased W/D ratio, MDA and MPO activity and decreased SOD activity. As shown in Table I, no significant differences were observed in the W/D ratio or the SOD, MDA and MPO activity of lung tissue among groups $\mathrm{A}, \mathrm{B}$ and $\mathrm{C}(\mathrm{P}>0.05)$, while LPS injection (groups D, E and F) caused a significant increase in the W/D ratio, MDA and MPO activity as compared with saline-injected rats (group $\mathrm{A})(\mathrm{P}<0.05)$, but with a significant decrease in SOD activity. These results demonstrated that oxidative damage appeared in lung tissue following LPS injection. No significant differences were observed in lung W/D ratios among groups $\mathrm{D}, \mathrm{E}$ and $\mathrm{F}(\mathrm{P}>0.05)$.

Sevo decreases lung tissue damage induced by LPS. As shown in Table I, compared with group F (only LPS injection), significant differences were observed in group D (Sevo post-conditioning) with increased SOD activity and decreased MDA and MPO activity. These results reflected that Sevo treatment significantly reversed the oxidant-antioxidant status and had a protective role. Compared with group D, LY294002 pretreatment led to a decrease in SOD activity and a slight increase in MDA and MPO activity; however, no significant difference was observed between groups $\mathrm{E}$ and $\mathrm{D}$.

Histological analysis and pathomorphological scores. Optical microscopic observation indicated the presence of intact alveoli structure, clean alveolar spaces, no alveolar interstititum, few inflammatory cell infiltrations and rare light alveolar wall thickening in groups A, B and C. However, in LPS-induced groups D, E and F, marked diffuse pulmonary edema of varying degrees, capillarectasia and hyperaemia were observed. In addition, margination and emigration of leucocytes widened alveolar spaces were also present, particularly in groups $\mathrm{E}$ and $\mathrm{F}$ (Fig. 2).

Pathomorphological scores were investigated and the results are shown in Table II. No significant differences were observed among groups $\mathrm{A}, \mathrm{B}$ and $\mathrm{C}(\mathrm{P}>0.05)$ and in comparison with these, pathomorphological scores were significantly increased in LPS-induced ALI models (groups D, E and F). However, compared with group F, pathomorphological scores 
Table II. Pathomorphological lung injury score in each group.

\begin{tabular}{lcccc}
\hline $\begin{array}{l}\text { Group } \\
(\mathrm{n}=8)\end{array}$ & $\begin{array}{c}\text { Congestion of } \\
\text { alveolar septae }\end{array}$ & $\begin{array}{c}\text { Intra-alveolar } \\
\text { cell infiltrates }\end{array}$ & $\begin{array}{c}\text { Alveolar } \\
\text { hemorrhage }\end{array}$ & $\begin{array}{c}\text { Total } \\
\text { score }\end{array}$ \\
\hline A & $0.13 \pm 0.35$ & 0 & 0 & $0.15 \pm 0.35$ \\
B & $0.25 \pm 0.46$ & 0 & 0 & $0.25 \pm 0.46$ \\
C & $0.25 \pm 0.46$ & 0 & 0 & $0.25 \pm 0.46$ \\
D & $2.13 \pm 0.64$ & $2.13 \pm 0.83$ & $2.13 \pm 0.64$ & $6.38 \pm 1.60^{\mathrm{a}}$ \\
E & $2.88 \pm 0.35$ & $2.88 \pm 0.35$ & $2.75 \pm 0.46$ & $8.50 \pm 1.07^{\mathrm{a}, \mathrm{b}}$ \\
F & $2.75 \pm 0.46$ & $2.88 \pm 0.35$ & $2.63 \pm 0.52$ & $8.13 \pm 0.64^{\mathrm{a}, \mathrm{b}}$ \\
\hline
\end{tabular}

${ }^{\mathrm{a} P}<0.05$, compared with A. ${ }^{\mathrm{b}} \mathrm{P}<0.05$, compared with D. A, control; B, $2.4 \%$ Sevo; C, LY294002 (phosphatidylinositol 3-kinase inhibitor); D, LPS + 2.4\% Sevo; E, LY294002 + LPS + 2.4\% Sevo; F, LPS.

A
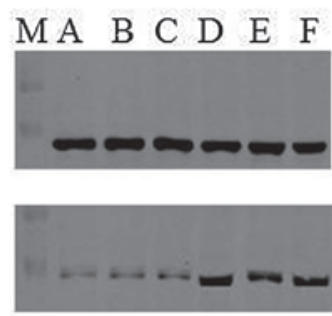

B

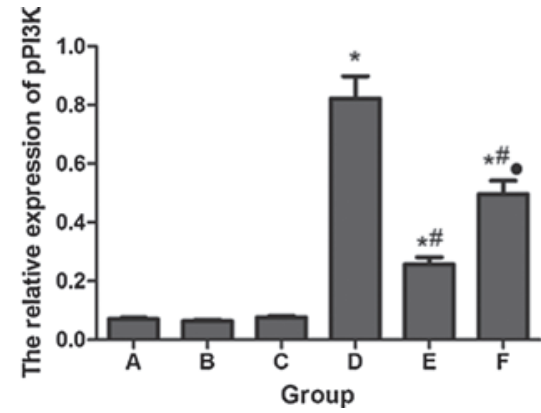

Figure 3. Protein expression levels of pPI3K in each group. (A) Western blot; (B) Quantification of A in a histogram. "P $<0.05$, compared with group A. "P $<0.05$, compared with group D. 'P $<0.05$, compared with group E. A, control; B, 2.4\% Sevo; C, LY294002 (phosphatidylinositol 3-kinase inhibitor); D, LPS + 2.4\% Sevo; E, LY294002 + LPS + 2.4\% Sevo; F, LPS; M, marker; pPI3K, phosphorylated phosphatidylinositol 3-kinase.

A
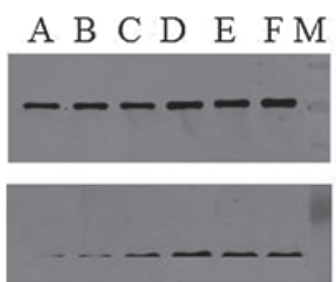

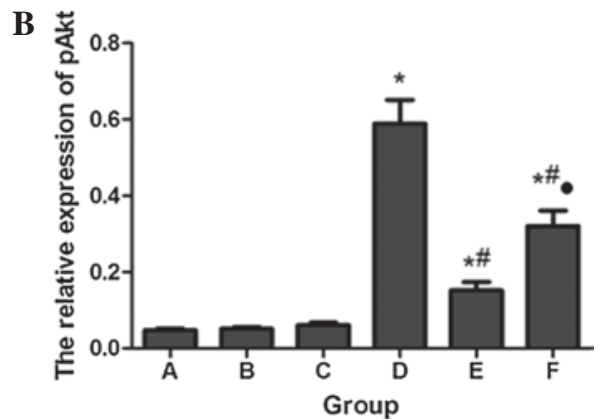

Figure 4. Protein expression levels of pAkt in each group. (A) Western blot; (B) Quantification of A in a histogram. "P $<0.05$, compared with group A. "P $<0.05$, compared with group D. 'P $<0.05$, compared with group E. A, control; B, 2.4\% Sevo; C, LY294002 (phosphatidylinositol 3-kinase inhibitor); D, LPS + 2.4\% Sevo; E, LY294002 + LPS + 2.4\% Sevo; F, LPS; M, marker; pAKT, phospho-Akt.
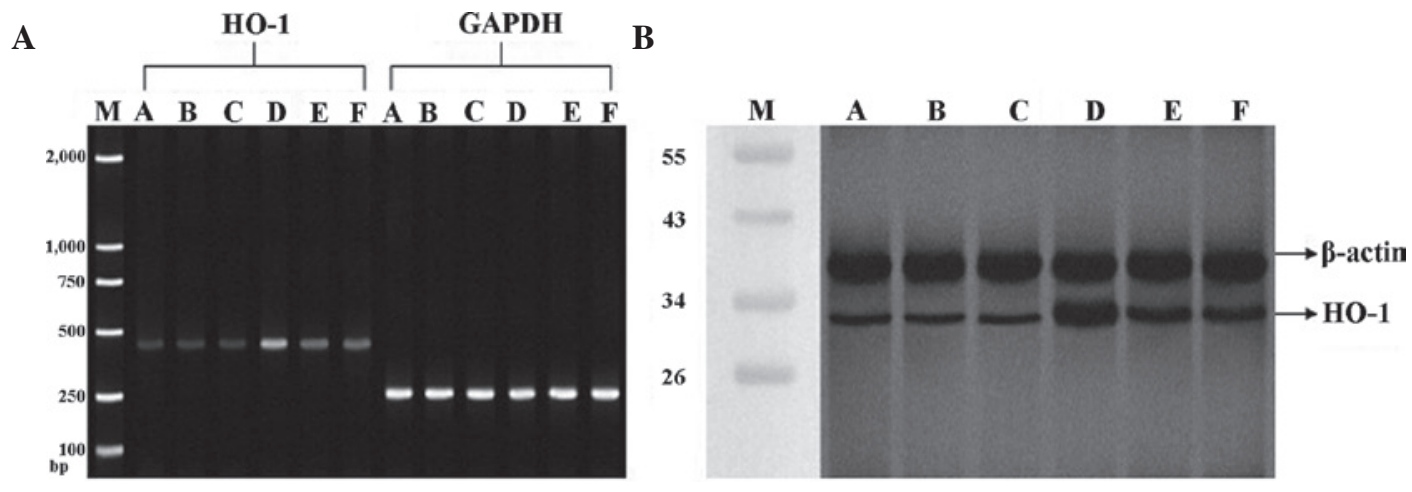

Figure 5. (A) mRNA and (B) protein expression levels of HO-1 in each group. A, control; B, 2.4\% Sevo; C, LY294002 (phosphatidylinositol 3-kinase inhibitor); D, LPS + 2.4\% Sevo; E, LY294002 + LPS + 2.4\% Sevo; F, LPS; M, marker. HO-1, oxygenase-1. 
in group $\mathrm{D}$ were significantly decreased $(\mathrm{P}<0.05)$ due to post-conditioning with $2.4 \%$ Sevo. Further analysis indicated that pathomorphological scores in group E were also significantly higher compared with those in group D, indicating that the PI3K inhibitor LY294002 eliminated the protective effects of Sevo.

Protein expression of $p P I 3 K$ and $p A k t$. The protein expression levels of pPI3K and pAkt in each group were detected by western blot analysis, using total PI3K and Akt as an internal standard. The results are shown in Figs. 3 and 4. There was limited pPI3K and pAkt expression in groups A, B and C, but a significant increase in group F. However, pPI3K and pAkt were further increased in group D following Sevo post-conditioning compared with group F. In addition, LY294002 pretreatment in group E significantly decreased pPI3K and pAkt expression compared with group D.

$m R N A$ and protein expression of $\mathrm{HO}-1$. As shown in Fig. 5, no significant differences were observed in mRNA and protein levels of HO-1 between groups A, B and C. LPS injection (groups D, E and F) induced a significant increase in mRNA and protein expression of $\mathrm{HO}-1$ as compared with group $\mathrm{A}$. A subsequent $2.4 \%$ Sevo inhalation (group D) significantly increased HO-1 expression compared with group F. Notably, HO-1 expression was significantly decreased in group E compared with group D, indicating that PI3K may be involved in the regulation of HO-1 expression.

\section{Discussion}

Inflammatory cells and inflammatory mediators have been well-characterized and are known to contribute to the overall pathogenesis of ALI. Infiltration with inflammatory cell has been found by histological analysis. Intercellular adhesion molecule (ICAM-1) is an inflammatory biomarker that is involved in the adhesion of monocytes to endothelial cells (ECs) and HO-1 has been reported to inhibit the expression of adhesion molecules in vascular ECs $(18,19)$.

At present, the expression of the HO-1 gene is primarily regulated at the transcriptional level, although there are variations between species (20). The human HO-1 gene is localized on chromosome $22 \mathrm{q} 12$ and consists of five exons and four introns, spanning $14-\mathrm{kb}(21,22)$. A promoter sequence is located 28 base pairs upstream from the starting site of transcription, which contains a number of enhancer and transcriptional regulatory elements, including the antioxidant response element (ARE), heat shock element and hypoxic response element. These transcriptional regulatory elements may specifically bind to a series of oxidative stress-responsive transcription factors (23), including nuclear factor- $\kappa \mathrm{B}$ (24), activator protein-1 (25) and nuclear factor erythroid 2-related factor (Nrf2) (26), suggesting a potential role of these factors in modulating $\mathrm{HO}-1$ induction.

Complex regulatory regions indicate the possibility that several signal transduction pathways may be involved in $\mathrm{HO}-1$ regulation, including mitogen-activated protein kinase, protein kinase $\mathrm{C}$ and tyrosine protein kinase $(27,28)$. A recent study indicated that the PI3K/Akt pathway also has a role in HO-1 regulation. For example, Chen et al (29) found that 4-ketopinoresinol, a novel naturally occurring ARE activator, induces activation of the $\mathrm{Nrf} 2 / \mathrm{HO}-1$ axis and protects against oxidative stress-induced cell injury via activation of PI3K/Akt signaling. PI3K, a lipid kinase, can be activated to generate the lipid second messenger PIP3 and subsequently activates its main downstream effector, the serine/threonine kinase Akt, which phosphorylates a variety of substrates that function to suppress apoptosis and promote progression through the cell cycle (30). A previous study found that Sevo post-conditioning activates the PI3K/Akt pathway to protect isolated rat hearts against I/R injury with decreased cardiomyocyte apoptosis (31). However, such cardioprotective effects were entirely eliminated by LY294002 (32). Similarly, post-conditioning with Sevo reduced nerve cell apoptosis, upregulated B-cell lymphoma 2 (Bcl-2) and downregulated P53 and Bcl-2-associated X protein. Wortmannin, a PI3K inhibitor, prevented pAkt from increasing, indicating that this neuroprotective effect may be partly due to the activation of the PI3K/Akt pathway and Akt phosphorylation (33).

In the present study, low levels of PI3K and pAkt were identified in the saline control groups A, B and C; however, expression significantly increased in the LPS-injection group. The activity of SOD was reduced, the activities of MDA and MPO were increased, and the mRNA and protein expression of HO-1 were all increased. This indicates that LPS elicited a marked oxidant stress response and activation of a protection mechanism.

However, Sevo post-conditioning reversed the oxidant damage status by increasing the SOD activity and decreasing MDA and MPO activity. In addition, treatment with Sevo also induced increasing HO-1 expression as well as PI3K and Akt phosphorylation. To confirm that the upregulation of $\mathrm{HO}-1$ expression was mediated by the PI3K/Akt pathway, the PI3K inhibitor LY294002 was introduced. The results showed that LY294002 inhibited PI3K and Akt phosphorylation, but also HO-1 expression at the mRNA and protein levels, indicating that the PI3K/Akt pathway may be involved in HO-1 expression induced by Sevo.

In conclusion, the present study suggested that the upregulation of HO-1 expression caused by inhalation of Sevo, which decreases the release of the inflammatory mediator ICAM-1 in ALI processes, may proceed via the PI3K/Akt pathway. Numerous advances in clinical applications of Sevo/HO-1 enhancers are expected in the coming years based on the present analysis.

\section{Acknowledgements}

This study was supported by the Hunan Provincial Science and Technology Plan Project of China (nos. 06SK3022 and 2013FJ4106) and the High Technology Industry Department, Development and Reform Commission of Hunan Province, China (no. 2012-1493).

\section{References}

1. Nakao A, Kaczorowski DJ, Zuckerbraun BS, Lei J, Faleo G, Deguchi K, McCurry KR, Billiar TR and Kanno S: Galantamine and carbon monoxide protect brain microvascular endothelial cells by heme oxygenase- 1 induction. Biochem Biophys Res Commun 367: 674-679, 2008

2. Kim HP, Ryter SW and Choi AM: CO as a cellular signaling molecule. Annu Rev Pharmacol Toxicol 46: 411-449, 2006. 
3. Mura M, Andrade CF, Han B, Seth R, Zhang Y, Bai XH, Waddell TK, Hwang D, Keshavjee S and Liu M: Intestinal ischemia-reperfusion-induced acute lung injury and oncotic cell death in multiple organs. Shock 28: 227-238, 2007.

4. Takemori K, Kobayashi K and Sakamoto A: Expression of pulmonary vasoactive factors after sevoflurane anaesthesia in rats: a quantitative real-time polymerase chain reaction study. Br J Anaesth 100: 190-194, 2008.

5. Schwer CI, Stoll P, Pietsch U, Stein P, Laqua J, Goebel U, Hoetzel A and Schmidt R: Up-regulation of heme oxygenase-1 by sevoflurane is not dependent on Kupffer cells and associates with ERK $1 / 2$ and AP-1 activation in the rat liver. Int J Biochem Cell Biol 42: 1876-1883, 2010.

6. Ye Z, Guo Q, Xia P, Wang N, Wang E and Yuan Y: Sevoflurane postconditioning involves an up-regulation of HIF-1alpha and HO-1 expression via PI3K/Akt pathway in a rat model of focal cerebral ischemia. Brain Res 1463: 63-74, 2012.

7. Osaki M, Oshimura M and Ito H: PI3K-Akt pathway: its functions and alterations in human cancer. Apoptosis 9: 667-676, 2004

8. Pachori AS, Smith A, McDonald P, Zhang L, Dzau VJ and Melo LG: Heme-oxygenase-1-induced protection against hypoxia/reoxygenation is dependent on biliverdin reductase and its interaction with PI3K/Akt pathway. J Mol Cell Cardiol 43: 580-592, 2007

9. OgborneRM,Rushworth SA and O'ConnellMA: Epigallocatechin activates haem oxygenase-1 expression via protein kinase Cdelta and Nrf2. Biochem Biophys Res Commun 373: 584-588, 2008.

10. Furukawa Y, Urano T, Minamimura M, Nakajima M, Okuyama S and Furukawa S: 4-Methylcatechol-induced heme oxygenase-1 exerts a protective effect against oxidative stress in cultured neural stem/progenitor cells via PI3 kinase/Akt pathway. Biomed Res 31: 45-52, 2010.

11. Park SY, Park da J, Kim YH, Kim Y, Kim SG, Shon KJ, Choi YW and Lee SJ: Upregulation of heme oxygenase-1 via PI3K/Akt and Nrf-2 signaling pathways mediates the anti-inflammatory activity of Schisandrin in Porphyromonas gingivalis LPS-stimulated macrophages. Immunol Lett 139: 93-101, 2011.

12. Sun Y, Cui Y and Wang J: Effects of sevoflurane on endotoxin-induced oxidative stress response of the lungs in rats Chin J Anesthesiol 8: 735-737, 2006 (In Chinese)

13. Crawford MW, Lerman J, Saldivia V and Carmichael FJ: Hemodynamic and organ blood flow responses to halothane and sevoflurane anesthesia during spontaneous ventilation. Anesth Analg 75: 1000-1006, 1992.

14. Coimbra R, Melbostad H, Loomis W, Porcides RD, Wolf P, Tobar M and Hoyt DB: LPS-induced acute lung injury is attenuated by phosphodiesterase inhibition: effects on proinflammatory mediators, metalloproteinases, NF-kappaB, and ICAM-1 expression. J Trauma 60: 115-125, 2006

15. Gao JZ, LV FH, Teng QL and Zhang JY: Role of adiponectin/phosphatidylinositol 3-kinase/protein kinase B signaling pathway on limb ischemic preconditioning on myocardial protection. Afr J Biotechnol 11: 10976-10980, 2012.

16. Al-Amran FG, Hadi NR and Hashim AM: Leukotriene biosynthesis inhibition ameliorates acute lung injury following hemorrhagic shock in rats. J Cardiothorac Surg 6: 81, 2011.

17. Itoh S, Taketomi A, Harimoto N, et al: Antineoplastic effects of gamma linolenic acid on hepatocellular carcinoma cell lines. J Clin Biochem Nutr 47: 81-90, 2010.

18. Yu AL, Lu CY, Wang TS, Tsai CW, Liu KL, Cheng YP, Chang HC Lii CK and Chen HW: Induction of heme oxygenase 1 and inhibition of tumor necrosis factor alpha-induced intercellular adhesion molecule expression by andrographolide in EA.hy926 cells. J Agric Food Chem 58: 7641-7648, 2010.
19. Chao CY, Lii CK, Tsai IT, Li CC, Liu KL, Tsai CW and Chen HW: Andrographolide inhibits ICAM-1 expression and $\mathrm{NF}-\kappa \mathrm{B}$ activation in TNF- $\alpha$-treated EA.hy926 cells. J Agric Food Chem 59: 5263-5271, 2011.

20. Kim HR, Kim S, Kim EJ, Park JH, Yang SH, Jeong ET, Park C, Youn MJ, So HS and Park R: Suppression of Nrf2-driven heme oxygenase-1 enhances the chemosensitivity of lung cancer A549 cells toward cisplatin. Lung Cancer 60: 47-56, 2008.

21. Loboda A, Jazwa A, Grochot-Przeczek A, Rutkowski AJ, Cisowski J, Agarwal A, Jozkowicz A and Dulak J: Heme oxygenase- 1 and the vascular bed: from molecular mechanisms to therapeutic opportunities. Antioxid Redox Signal 10: 1767-1812, 2008.

22. Funke C, Tomiuk J, Riess O, Berg D and Soehn AS: Genetic analysis of heme oxygenase-1 (HO-1) in German Parkinson's disease patients. J Neural Transm 116: 853-859, 2009.

23. Chung HT, Pae HO and Cha YN: Role of heme oxygenase-1 in vascular disease. Curr Pharm Des 14: 422-428, 2008.

24. Choi HG, Lee DS, Li B, Choi YH, Lee SH and Kim YC: Santamarin, a sesquiterpene lactone isolated from Saussurea lappa, represses LPS-induced inflammatory responses via expression of heme oxygenase- 1 in murine macrophage cells. Int Immunopharmacol 13: 271-279, 2012.

25. Soo Kim H, Young Park S, Kyoung Kim E, Yeon Ryu E, Hun Kim Y, Park G and Joon Lee S: Acanthopanax senticosus has a heme oxygenase-1 signaling-dependent effect on Porphyromonas gingivalis lipopolysaccharide-stimulated macrophages. J Ethnopharmacol 142: 819-828, 2012.

26. Harada H, Sugimoto R, Watanabe A, et al: Differential roles for Nrf2 and AP-1 in upregulation of HO-1 expression by arsenite in murine embryonic fibroblasts. Free Radic Res 42: 297-304, 2008.

27. Wang L, Kou MC, Weng CY, Hu LW, Wang YJ and Wu MJ: Arsenic modulates heme oxygenase-1, interleukin-6, and vascular endothelial growth factor expression in endothelial cells: roles of ROS, NF- $\mathrm{B}$, and MAPK pathways. Arch Toxicol 86: 879-896, 2012.

28. Wu Y, Dong Y, Song P and Zou MH: Activation of the AMP-activated protein kinase (AMPK) by nitrated lipids in endothelial cells. PLoS One 7: e31056, 2012.

29. Chen HH, Chen YT, Huang YW, Tsai HJ and Kuo CC: 4-Ketopinoresinol, a novel naturally occurring ARE activator, induces the Nrf2/HO-1 axis and protects against oxidative stress-induced cell injury via activation of PI3K/AKT signaling. Free Radic Biol Med 52: 1054-1066, 2012.

30. Graham JR, Hendershott MC, Terragni J and Cooper GM: mRNA degradation plays a significant role in the program of gene expression regulated by phosphatidylinositol 3-kinase signaling. Mol Cell Biol 30: 5295-5305, 2010.

31. Zheng Z, Yang M, Zhang F, Yu J, Wang J, Ma L, Zhong Y, Qian L, Chen G, Yu L and Yan M: Gender-related difference of sevoflurane postconditioning in isolated rat hearts: focus on phosphatidylinositol-3-kinase/Akt signaling. J Surg Res 170: e3-e9, 2011.

32. Yao YT, Fang NX, Shi CX and Li LH: Sevoflurane postconditioning protects isolated rat hearts against ischemia-reperfusion injury. Chin Med J (Engl) 123: 1320-1328, 2010.

33. Wang Y, Romigh T, He X, Orloff MS, Silverman RH, Heston WD and Eng C: Resveratrol regulates the PTEN/AKT pathway through androgen receptor-dependent and -independent mechanisms in prostate cancer cell lines. Hum Mol Genet 19: 4319-4329, 2010. 